\title{
New vista in magnetic modulation of light scattering by magnetic spheres in magnetic medium
}

\author{
Hem Bhatt \\ Shantilal Shah Engineering College, Sidsar, Bhavnagar. 364060, Gujarat, India \\ Email: drhembhatt@gmail.com
}

Light scattering is one of such realm of research which is explored a lot in recent past by many scientists because of wide range of applications in nano-photonic devices and molecular sensors in the field of biology, medical sciences, energy sources, clocked tools, perfect lenses, etc. Mie resonances produced by magnetic and non magnetic spheres in magnetically passive medium are already studied for various size parameters and different optical properties. Here we have studied effect of magnetic field on micron sized magnetic spheres on Mie coefficients, when dispersed in magnetically active medium. Recently some researchers have studied light scattering by magnetic particle in magnetically active medium. Mie coefficients are basic parameters to study forward and back scattered intensity of scattered light, scattering cross section scattering efficiency, etc. Mie coefficients $a_{n}$ and $b_{n}$ are studied as a function of magnetic field.

We consider a magnetizable sphere of arbitrary size parameter and refractive index $m_{s}$ surrounded by a magnetizable medium like a ferrofluid. When the spheres are irradiated by plane waves the scattering intensity and scattering efficiency can be studied in terms of external Mie coefficients $\left(a_{n}\right.$ and $\left.b_{n}\right)$ and internal Mie coefficients $\left(c_{n}\right.$ and $\left.d_{n}\right)$. As the surrounding medium is magneto-optically active, it's refractive index changes when subjected to external magnetic field. This in turn affects refractive index of the micron sized magnetic scatterer, consequently modulating scattering. The field dependent refractive index of magnetic fluid can be given as $m_{0}+$ $m_{\infty} L(\alpha)$, where $m_{0}$ is refractive index of the medium in absence of external magnetic field and $m_{\infty}$ is saturation refractive index in presence of external magnetic field. $\mathrm{L}(\alpha)=$ Langevin's function with argument $\alpha$ and $\alpha$ is ratio of magnetic potential energy of the nano-magnetic particles of the medium due to external magnetic field and thermal energy due to temperature. The refractive index of magnetic sphere with respect to medium can be given as ratio of absolute refractive index of the magnetic sphere to the field dependent refractive index of surrounding medium (i.e. magnetic fluid).

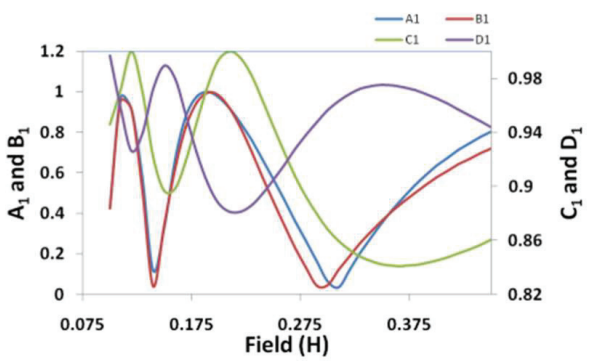

Figure 1: External and internal Mie coefficients as a function of magnetic field for scatterer size $7 \mu \mathrm{m}$

Figure 1 shows the external and internal Mie coefficients as a function of magnetic field for a scatterer size $7 \mu \mathrm{m}$. We show here that with change in external magnetic field Mie coefficients exhibit resonances indicating modulation in scattering as well as internal field of scatterer. Significance of the present study evidences from the fact that Mie coefficients are primary parameters to study scattering phenomenon. The resonance noticed in sets of Mie coefficients $\left(a_{1}, b_{1}\right)$ and $\left(c_{1}, d_{l}\right)$ are indicators to the variation in scattered intensity and internal electro-magnetic field as well as energy stored inside magnetic spheres. This opens a new field in scattering phenomenon, which has been found interesting due to multitude of applications in magneto-optics and magneto-photonics, by means of magnetic field.

\section{References}

1. Bohren C. and D. R. Huffman, Absorption and Scattering of Light by Small Particle, Johm Wiley, New York, NY (1983)

2. Abramowitz M. and I. A. Stegun (eds.), Handbook of Mathematical Functions, Dover Publication,New York, NY (1965). 